\title{
Combined oral contraception and cancer
}

Jacqui Tuckey, MB ChB, MRCOG, DFFP, Specialist Registrar in Community Gynaecology, Ella Gordon Unit, St Mary's Hospital, Milton Road, Portsmouth PO3 6AD, UK

(Accepted August 24th 2000)

\section{How to use a FACT}

A FACT is an up-to-date review of a subject relevant to the speciality, intended to help you fulfil your CPD requirements in your home or place of work. Whilst FACTs are edited and reviewed at various levels within the Faculty, the actual contents and views expressed are those of the authors and not the Faculty. More specifically, these reviews are not guidelines. The CEC is producing clinical guidelines separately.

FACTs have three sections: a review, a true/false test, and discussion points. To use a FACT to earn CPD credits you should do the following:

1. Working alone: Read the review and do the test. The answers are provided on page 247 so you can mark yourself. If there are points you are unsure about, disagree with, or need further clarification on, make a note of these for use at a later date. This should take you no more than 1 hour. Keep a record of having done this in your CPD diary and, unless indicated otherwise on the FACT, this will earn you 1 hour (DFFP), 1 credit (MFFP).

2. Working as a group: arrange a meeting of at least 1 hour with colleagues to discuss the discussion points given in the FACT (page 240) and any issues the participants have come up with as a result of reading the FACT. Keep a record of having done this in your CPD diary and, unless indicated otherwise on the FACT, this will earn you 1 hour (DFFP), 1 credit (MFFP).

\section{Introduction}

Since their introduction in the 1960s the safety of combined oral contraceptives (COCs) has been widely debated. The difficulty in determining if $\mathrm{COC}$ use increases the risk of developing cancer is due to:

- the long latent period between exposure to the potential carcinogen and overt malignant disease

- the fact that due to the developments in COCs women are likely to have taken a variety of different pills

- the paucity of data on the use of COCs in women over the age of 35 .

\section{Breast cancer}

The association between breast cancer and oral contraceptive use is rapidly becoming one of the most investigated areas of epidemiology. Epidemiologists have long been aware of the link between exposure to endogenous ovarian hormones and breast cancer: late menarche, early menopause and oophorectomy being associated with a decreased risk, linked to a reduced lifetime exposure to oestrogen and progesterone.

The issue of COCs and breast cancer has always been contentious. Several epidemiological studies have suggested that COCs may increase the risk of breast cancer, but many lacked the statistical power to determine the exact risk. In 1996, the Collaborative Group on Hormonal Factors in Breast Cancer published the results of a reanalysis of world-wide epidemiological studies on this subject. ${ }^{1}$ It included data on 53297 cases and 100239 controls from 54 studies in 25 countries - an estimated $90 \%$ of all existing data on COC and breast cancer. Even this study had insufficient data on the use of COC by older women and also on long-term use.

The findings of this study were:

- an increased relative risk of 1.24 of having breast cancer diagnosed whilst on any COC, decreasing during the 10 years after stopping.

- the disappearance of any increased risk, compared to non-users, 10 years after stopping the COC. 
- cancers in users were less clinically advanced, but there were no data on mortality

- a family history of breast cancer, duration of use, age at first use and the dose and type of hormone had no additional effect on the risk of having breast cancer once length of time since last use had been taken into account.

Table 1 The relative risk of having breast cancer diagnosed in relation to COC use

\begin{tabular}{lcc}
\hline COC Usage & Relative risk & Significance \\
\hline Current users & 1.24 & $\mathrm{P}<0.00001$ \\
1-4 years after stopping & 1.16 & $\mathrm{P}<0.00001$ \\
5-9 years after stopping & 1.07 & $\mathrm{P}<0.009$ \\
10+ years after stopping & 1.01 & Not significant \\
\hline
\end{tabular}

Source: Faculty of Family Planning statement on breast cancer 1996, adapted from reference 1 .

Table 1 shows the relative risk of having breast cancer while taking the $\mathrm{COC}$ and in the 10 years after stopping. The risk of breast cancer increases as women grow older whether they use COC or not. The incidence of breast cancer by age is shown in Table 2 .

Table 2 The cumulative risk of breast cancer by age

\begin{tabular}{ccc}
\hline By age 35 & By age 45 & By age 75 \\
\hline 1 in 500 & 1 in 100 & 1 in 12 \\
\hline
\end{tabular}

Table 3 shows the excess number of breast cancer cases per 10000 women who had used COCs for 5 years and who were followed up for 10 years after stopping (compared with never users of the COC).

Table 3 The estimated excess risk of breast cancer per 10000 women using the COC for 5 years, by age

\begin{tabular}{lllllll}
\hline COC use for 5 years to age... 20 & 25 & 30 & 35 & 40 & 45 \\
\hline Excess cases per 10000 & 0.5 & 1.5 & 5 & 10 & 20 & 30
\end{tabular}

The incidence of breast cancer in women under 40 is low, therefore the absolute increased risk in young women is very small. Because of the increase in incidence of breast cancer with age (Table 2), the attributable risk increases in older women.

The authors of this study did postulate that the same effect was possible with other hormonal contraceptives, but in this reanalysis the risks for progestogen-only contraceptives, which showed a similar trend to that for COCs, were not statistically significant. This failure to demonstrate significance may be due to the small number of subjects in the study using progestogen-only methods.

\section{Ovarian and endometrial cancer}

Because the COC prevents repeated stimulation of the ovary and endometrium, it is postulated that it reduces the risk of malignancy. This protective effect has been well established in studies of pills containing greater than $30 \mu \mathrm{g}$ of ethinyloestradiol. In a rare consensus of opinion all casecontrol and cohort studies agree.

\section{Cancer of the ovary}

Ovarian cancer is the most common fatal gynaecological malignancy with a 5 year survival rate of around $40 \%$. The lifetime risk of developing ovarian cancer for women in developed countries is $2 \%$. Little is known about the aetiology, but incidence increases with age and family history and risk is reduced with parity and COC use. Table 4 shows the incidence of both ovarian and endometrial cancers per 100000 women in England and Wales for 1992.

Table 4 Incidence of endometrial/ovarian cancer per 100000 women in England and Wales (1992)

\begin{tabular}{lcc}
\hline Age & Endometrial cancer & Ovarian cancer \\
\hline $15-34$ & 0.3 & 1.9 \\
$35-54$ & 10.4 & 18.7 \\
$55-64$ & 40.4 & 49.2 \\
$65-74$ & 45.6 & 59.0 \\
$75-84$ & 45.0 & 58.0 \\
\hline
\end{tabular}

Source: ONS National Cancer Registration Database

A Collaborative analysis of 12 case-control studies conducted in the US 1956-86 looked at data for 2197 cases of invasive ovarian epithelial cancer and 8893 controls. $^{2}$ The findings were that women who had ever used COC had an odds ratio of 0.7 for invasive ovarian cancer compared with never users. With increasing years of use the risk decreases, but there is little additional benefit after 6 years of use. Most data have suggested that the protective effect persists for at least 15 years; this analysis found that risk of cancer decreased with increased time since last use. (Women who had used the COC in the distant past, i.e. 15 years or more prior to the reference date, were at lower risk than recent users. This may be because they were more likely to have used higher dose preparations). How much of this protective effect can be expected for women taking COCs containing less than $50 \mu \mathrm{g}$ ethinyloestradiol has not been established.

The increase in use of the COC over the last 30 years has been paralleled by a decline in the incidence of ovarian cancer. ${ }^{3}$ The protection offered by the COC is valuable given the poor survival rate from the disease.

\section{Cancer of the endometrium}

Numerous case-control studies have demonstrated a protective effect of the $\mathrm{COC}$ in relation to endometrial cancer. The effect seems to be mediated through progestogenic opposition to oestrogenic stimulation of the endometrium. The most detailed information has come from the Cancer and Steroid Hormone Study (CASH ). This reported a halving of risk of endometrial cancer for $\mathrm{COC}$ users compared to non-users. ${ }^{4}$ There was no significant effect if the COC was used for less than 1 year, but with more than 1 year of use the effect persisted for at least 15 years after stopping the COC. Once again it is not established how much of this protective effect can be expected for women taking COCs containing less than $50 \mu \mathrm{g}$ ethinyloestradiol. A global downward trend in mortality from endometrial cancer has been noted and the COC may have a contributory role. ${ }^{5}$

\section{Cancer of the cervix}

Cancer of the cervix is the eighth most common cancer in women and the most common cancer in women under 35 years. Ninety percent of cases are squamous cell carcinoma, the remaining $10 \%$ are adenocarcinomas. The incidence is approximately $15 / 100000$ and overall 5 year survival is $60 \%$. Incidence peaked in 1988 and has steadily declined subsequently; this may be associated with improvements made to the NHS Cervical Screening Programme.

A World Health Organization (WHO) collaborative study was initiated in 1979 to determine whether risks of breast, 
hepatobiliary, endometrial, ovarian and cervical cancers are altered by the use of steroid contraceptives. The study reported findings related to invasive squamous cell carcinoma of the cervix in 1993.6 The investigators found a relative risk of 1.31 for cervical cancer in women who had ever used the COC; this would result in 5 extra cases per 100000 women per year. No significant increase in risk was observed until over 5 years from first use, suggesting a latent period. Risk declined after cessation of use to that of non-users by 8 years.

The Royal College of General Practitioners (RCGP) Oral Contraception Study showed an excess of cases of invasive cervical carcinoma of eight per 100000 women years in COC users compared to non-users. ${ }^{7}$ They found that the incidence of cervical cancer increases with increased duration of COC use. The incidence of cervical cancer is influenced by sexual behaviour. However, both the WHO and RCGP studies controlled for some of the relevant confounding factors. The cause of cervical cancer is not known with certainty, but human papilloma virus (HPV), is increasingly recognised as the major aetiological agent and, in addition, smoking is estimated to double a woman's risk of developing the disease.

Most epidemiological studies have found an association between risk of cervical carcinoma in situ and COC use, but some studies have not and it remains uncertain whether there is a causal relationship. ${ }^{8}$

Adenocarcinoma of the cervix constitutes $10 \%$ of all cervical cancers. In the USA from the early 1970s to the mid-1980s the incidence more than doubled in women under 35 years; similar increases were reported in the UK. This increase is partly due to improved pathological diagnosis. Ever users of oral contraception are twice as likely to develop the disease as never users, and this rises to four times as likely with use for longer than 12 years. ${ }^{9}$ This work is subject to the same confounding as the work on squamous cell disease.

All young women should be encouraged to have regular cervical smears as recommended by the Cervical Screening Programme. In the light of the above data it would appear to be particularly important for long-term COC users to have regular routine cytology according to the NHS Cervical Screening Programme.

\section{Liver cancer}

Hepatocellular adenoma is a non-malignant liver tumour, which became more prevalent after the introduction of COC in the 1960 's. ${ }^{10}$ Risk has been correlated to dose and duration of use. However, it is a very rare disease and the attributable risk has been estimated to be no more than three per 100000 COC users under the age of 30 per year. $^{11}$

Five out of six case-control studies of hepatocellular carcinoma (HCC) and COC use published since 1983 have shown an elevated relative risk of four; again risk is strongly dependent on duration of use. The absolute risk remains very small because the disease is so rare - a maximum of 12 cases per year in England and Wales would be attributable to long-term COC use. ${ }^{12}$ Because of the low absolute risk of developing this condition, the fact that it may occur is not usually an important factor to consider when deciding to prescribe or use COC. The studies are of women using $50 \mu \mathrm{g}$ pills, and it may be that modern low dose pills confer less risk.

\section{Malignant melanoma}

The possible role of exogenous oestrogens in the aetiology of malignant melanoma has been controversial since the 1970s. Several conclusions are possible with the current state of knowledge. Firstly, there is no evidence that exogenous oestrogens play a part in the aetiology of melanoma. Secondly, women have a survival advantage over men, which could be due to endogenous oestrogens inhibiting melanoma growth. Thirdly, exogenous oestrogens do not promote disease progression. Therefore women with a history of melanoma can safely use COC. ${ }^{13}$ However, it is still prudent to have a full discussion with, and the agreement of, the oncologist.

\section{Choriocarcinoma}

Current guidelines advise that the COC, if taken before the return to normality of human chorionic gonadotrophin (hCG) values following hydatidiform mole, may increase the need for treatment. They should therefore be avoided until hCG, as tested by a specialist laboratory, is undetectable in serum. ${ }^{14}$

\section{Colorectal cancer}

It has been suggested that cancer of the colon may share common aetiological factors with cancers of the breast and genital tract in women. It is postulated that oral contraceptives decrease the risk of colon cancer by reducing bile acid synthesis. Data from the Nurses Health Study in the United States were reported showing a significant reduction of colorectal cancer in women who had used $\mathrm{COC}$ for 8 years or more (relative risk 0.60 compared with never users). ${ }^{15}$ It is not established if any protective effect can be expected for women taking COCs containing less than $50 \mu \mathrm{g}$ ethinyloestradiol.

\section{Risks and benefits}

COCs appear to promote some cancers and protect against others. Current users have an increased relative risk of breast cancer, which diminishes to the background rate over the 10 years after stopping; no long-term increase in risk has been shown in former users. The increase in actual risk of breast cancer with age means that the attributable risk is most significant in women over 35 years taking COC. In young women, the increase in relative risk translates into very small numbers of excess cases. It is particularly reassuring that breast cancer appears to be less advanced in COC users than non-users.

There is an increased risk of cervical cancer with increased duration of COC use; this is more marked with adenocarcinoma than squamous carcinoma, but adenocarcinoma constitutes only $10-15 \%$ of cases.

Case-control studies have demonstrated an increased relative risk of liver cancer for ever users of oral contraception. The incidence of liver cancer in the UK population is, however, extremely small.

Case-control studies have shown a protective effect of ever use of COC against endometrial and ovarian cancer with $50 \mu \mathrm{g}$ ethinyloestradiol pills, which is long lasting after discontinuation. It is not known to what extent this protective effect is present for women taking COCs containing less than $50 \mu \mathrm{g}$ ethinyloestradiol. Breast cancer is five times more common than ovarian cancer, but both affect women of similar ages. Endometrial cancer is less common and is unusual in women under 50 years.

\section{Conclusion}

From a population perspective the cancer related risks and benefits associated with COC use are small and the net overall effect is likely to be negligible. For the majority of women the advantages of oral contraception outweigh the 
disadvantages from uncommon but serious long-term complications.

References

1 Collaborative Group on Hormonal Factors in Breast Cancer. Breast cancer and hormonal contraceptives. Lancet 1996; 347: 1713-727.

Whittemore AS, Harris R, Itnyre J and the Collaborative Ovarian Cancer Group. Characterstics relating to ovarian cancer risk: collaborative analysis of 12 US case-control studies. American

gy of epithelial ovarian cancer: a review. Int $J$ Cancer and Steroid Hornor

mones (CASH) 1987a. Combined oral contraceptive use and risk of endometrial cancer. JAMA 1987; 257: 796-800.

and risks of oral contraceptives. Advances in Contraception 1990; 6: 15-25.

WHO Collaborative Study. Invasive squamous-cell carcinoma and combined oral contraceptives: results from a multinational study. Int J Cancer 1993; 55:228-36.
Beral V, Hannaford P, Kay C. Oral contraceptive use and malignancies of the genital tract Lancet $1988 ; 2: 1331-335$.

Ye Z, Thomas DB, Ray RM and the WHO Collaborative Study of Neoplasia and Steroid Contraceptives. Combined oral contraceptives and risk of cervical carcinoma in situ. Int $J$ Epid 1995; 24:19-26.

(3): $1390-394$, Urin G, Peters RK, Henderson BE,

Thoro Lancet 1994, 334. 1390-394. Thorogood M. Combined oral contraceptives. Tisks and benefits. Br Med Bulletin 1993; 49:

Rosenberg L. The risk of liver neoplasia in relation to combined oral contraceptive use. (

D, Doll R, et al. Oral contraceptives and hepatocellular carcinoma. $B M$

RCOG Guidel Sex, pregnancy, hormones, and melanoma. $B M J 1993 ; 307: 2-3$. RCOG Press, April 1999.

Martinez ME, Grodstein F Giovannucci E et al. A prospective study of reproductive factors, oral contraceptive use and risk of colorectal cancer. Cancer Epidemiology 1997; 6: 1-5.

\section{Discussion points}

1. What advice should we give to young women with a family history of breast cancer who have concerns regarding use of the COC?

2. Consider the important concepts of absolute, relative and attributable risks.

3. Should women with cervical intraepithelial neoplasia be advised that they may continue to use the COC, or should they consider an alternative contraceptive method?

4. How much information is it necessary to give to COC users regarding cancer risks?

F Faculty

A Aid to

A CPD Self-Assessment Test

C CPD

T Topics

QUESTION SHEET

Review No. 2000/04

To be reviewed not later than 31 August 2005

\section{Combined oral contraception and cancer}

Indicate your answer by ticking the appropriate box for each question

True

False

1. Late menarche/early menopause increase the risk of breast cancer.

2. Within 10 years of stopping the COC the risk of breast cancer is the same as that of non-users.

3. Breast cancers diagnosed in COC users tend to be less clinically advanced

4. A family history of breast cancer has a synergistic effect with COC use.

5. The protective effect of higher dose COC use on ovarian cancer appears to persist for at least 15 years.

6. Use of the COC may have contributed to a global upward trend in mortality from endometrial cancer.

7. Smoking halves a woman's risk of cervical cancer.

8. There is an increased risk of cervical cancer with increased duration of COC use.

9. The increased risk of hepatocellular carcinoma should always be discussed with a woman starting the COC.

10. Following hydatidiform mole, hormonal contraceptives should be avoided until hCG is undetectable in serum as measured by a specialist laboratory. 